\title{
Cryptococcal meningitis with concurrent pulmonary cryptococcosis: Retrospective clinical analysis of 12 cases
}

\author{
Zhu-qin Liu*, Jue Hu, Hui Xiao and Li-xin Huang \\ Neurology Department, Central Hospital of Changsha City, Changsha 410004, China.
}

Accepted 3 April, 2012

\begin{abstract}
The clinical and imaging characteristics of cryptococcal meningitis (CM) with concurrent pulmonary cryptococcosis (PC) were presented in the present study. Retrospective clinical data analysis of 20 histopathologically confirmed cases of CM with concurrent PC. The patients included eight males and four females, with four 62-year olds and an average age of 33.3 years \pm 23.8 years. The course was from 14 days to 120 days $(41.0 \pm 39.9)$. The first symptom in ten cases was fever, eight cases had headache, three cases had productive cough and one case had chest pain. Ten cases have underlying diseases, which include six cases of chronic viral hepatitis B, three cases of silicosis with concurrent tuberculosis, one case of malnutrition, one case of systemic lupus erythematosus and one case of sinusitis. Ten of the cases were misdiagnosed as pulmonary tuberculosis, and one case was misdiagnosed as lung cancer. The chest X-rays revealed patchy, nodular shadows scattered in both lungs, with blurred edges and irregular low density areas. The computed tomography (CT) showed highly dense, patchy and speckled shadows widespread in both lungs, cavities of varying sizes, and elliptical, smooth-edged high density nodules. The cases were treated with intravenous amphotericin B (AMB) combined with fluconazole and intrathecal AMB. Eight cases were cured, whereas four cases improved. The clinical symptoms and imaging of CM with concurrent PC lack specificity. The increasing the understanding of $\mathrm{CM}$ imaging is beneficial to early diagnosis.
\end{abstract}

Key words: Cryptococcus neoformans meningitis, pulmonary cryptococosis, imaging.

\section{INTRODUCTION}

Cryptococcosis is one of the most common complications of acquired immunodeficiency syndrome (AIDS) among patients overseas. In recent years, the incidence of cryptococcosis is rising in China (Lai et al., 2005). The probable causes are the increasing incidence of chronic consumptive conditions, such as tuberculosis, cancer, diabetes mellitus, AIDS and organ transplantation among others (Lai et al., 2005; Wei and Li, 2007; Xu et al., 2011). These conditions can cause reduced immunity which are easily complicated with cryptococcosis. Secondly, the widespread use of immunosuppressants,

*Corresponding author. E-mail: liuzhuqincn@126.com. glucocorticoid, anticancer agents and broad-spectrum antibiotics destroys the human immune system and induces cryptococcal infections (Lai et al., 2005; Wei and $\mathrm{Li}, 2007$; Xu et al., 2011). Cryptococcal meningitis (CM) is an infectious disease of central nervous system caused by Cryptococcus neoformans. Considering the high risk of misdiagnosis and mistreatment in its early stages and the lack of effective medical treatment in latter stages, the mortality of CM is very high (Liu et al., 2002; Hu et al., 2006). Pulmonary cryptococcosis (PC) is a lung mycosis caused by $C$. neoformans. Given the lack of specificity of its clinical symptoms and low sensitivity of sputum analysis, PC is usually clinically misdiagnosed as lung cancer, nonspecific lung inflammation, tuberculosis and so on (Liu et al., 2002; Lai et al., 2005; Hu et al., 2006; 


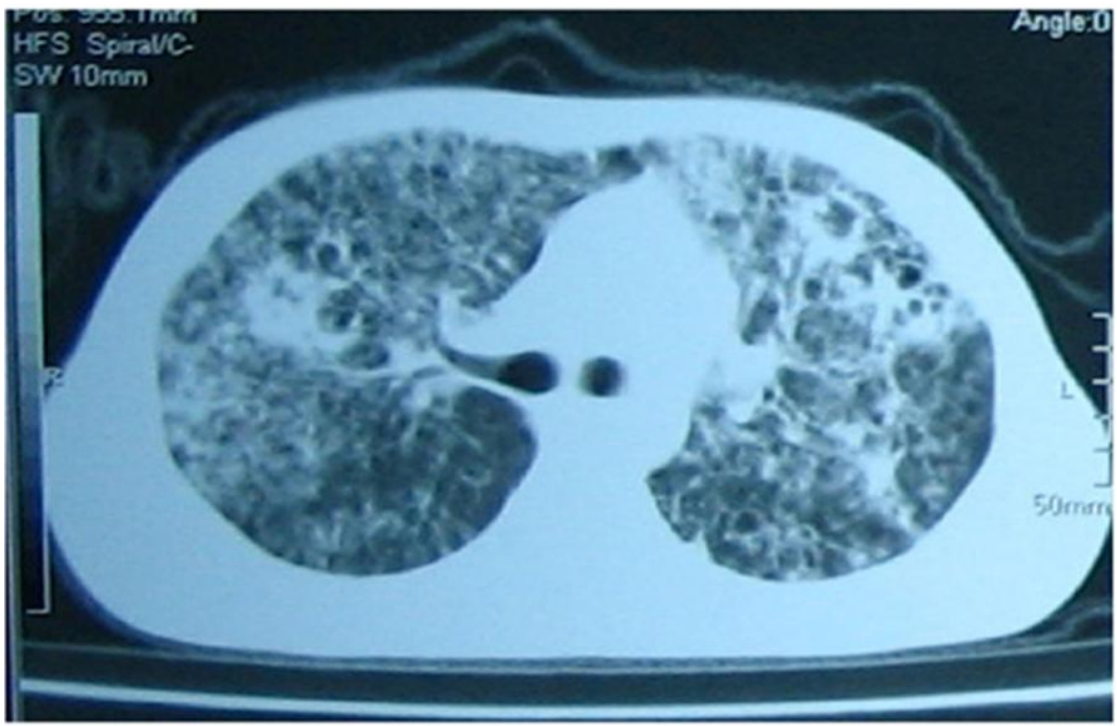

Figure 1. CT shows widespread patchy and speckled increased density shadows and cavities varying in size. The left lower lobes of lungs had more.

Wei and Li, 2007; Xu et al., 2011). A deep understanding of this disease is necessary for early diagnosis and improvement of the cure rate. Cases of $\mathrm{CM}$ with concurrent PC are rarely reported; therefore, a retrospective analysis of the clinical and imaging data of 12 cases treated in Central Hospital of Changsha City, China from January 2002 to August 2010 was done to increase the understanding of this disease.

\section{PATIENTS AND METHODS}

\section{Patients}

A total of $12 \mathrm{CM}$ cases with reliable data were diagnosed and treated in the hospital from January 2002 to August 2010. The patients include eight males and four females, with four 62-year old patients, and an average age of 33.3 years \pm 23.8 years. The course was from 14 to 120 days $(41.0 \pm 39.9)$. Ten of the cases had underlying diseases, which included six with chronic viral hepatitis $\mathrm{B}$, four with concurrent pneumosilicosis and tuberculosis, one with malnutrition, one with systemic lupus erythematosus (SLE), and one with sinusitis. All patients have negative HIV antibodies. Ten cases were misdiagnosed as tuberculosis infection, and one case was misdiagnosed as lung cancer.

\section{Clinical manifestation}

Nine cases were classified as subacute onset, whereas the remaining three cases were classified as chronic onset. The first symptoms in ten cases were fever, eight had headache, three had productive cough, and one case of chest pain. In addition to the symptoms given previously, two experienced vomiting, three had altered consciousness, one had abdominal pain, two had blurred vision, two had hearing impairment, and two developed brain herniation. Ten cases had neck rigidity, and six showed positive Kernig's sign, and two cases were positive for Babinski's sign.

\section{Laboratory examinations}

C. neoformans was detected in the cerebrospinal fluid of all 12 cases. India ink staining was positive in 11 cases. C. neoformans was found in the cerebrospinal fluid culture of 12 cases, with an initial positive rate of $40 \%$. The cerebrospinal spinal fluid was tested, which revealed the following results: pressure, 210 to $600 \mathrm{mmH}_{2} \mathrm{O}\left(362.0 \pm 134.7 \mathrm{mmH}_{2} \mathrm{O}\right)$; protein content, 0.29 to $2.40 \mathrm{~g} / \mathrm{l}(0.88 \pm 0.56 \mathrm{~g} / \mathrm{l})$; chloride content, 77 to $132 \mathrm{mmol} / \mathrm{l}$ $(110.2 \pm 14.1 \mathrm{mmol} / \mathrm{l})$; and sugar content, 0.32 to $4.07 \mathrm{mmol} / \mathrm{l}$ $(2.08 \pm 1.24 \mathrm{mmol} / \mathrm{l})$. The white blood cell count was 8 to 550 $(129.9 \pm 181.5) \times 10^{6} /$ l. Lymphocytes were predominant in the cell analysis. $C$. neoformans were detected from the specimen smears of the purulent liquid aspirated from a focus in the lungs or during the specimen culture of one case. A total of 11 cases were diagnosed based on the specimen smear from the respiratory tract or during the culture of the specimen. The pathologic examination confirmed the presence of $C$. neoformans in the lymph node biopsy from one case.

\section{Imaging examination}

The lung CT showed widespread patchy and speckled dense shadows and cavities of varying sizes in eight cases. The cavities were predominantly found in the lower lobes of the lungs, with cavities measuring up to $42 \mathrm{~mm} \times 42 \mathrm{~mm}$. The grain of both lungs was increased and disordered, and the interlobular septa were greatly thickened and showed a reticular change (Figures 1 and 2). The mediastinal lymph nodes were enlarged; the nodes adjacent the trachea and aorta were enlarged in 2 cases. The left pleural cavity was thickening with adhesion in one case. The lung CT of five cases showed $6 \mathrm{~mm}$ to $15 \mathrm{~mm}$ highly dense, non-homogenous nodules, with elliptical, smooth edges (Figure 3). The chest X-ray showed patchy, nodular, miliary shadows scattered in both lungs, and blurred edges, scattered along the lung grain, with nonhomogenous densities. Some foci coalesced into clump foci, with irregular low density areas (Figures 4 and 5). The antifungal treatment was effective based on the lung imaging studies. The lesions were reduced or disappeared. Ten cases had cranial 


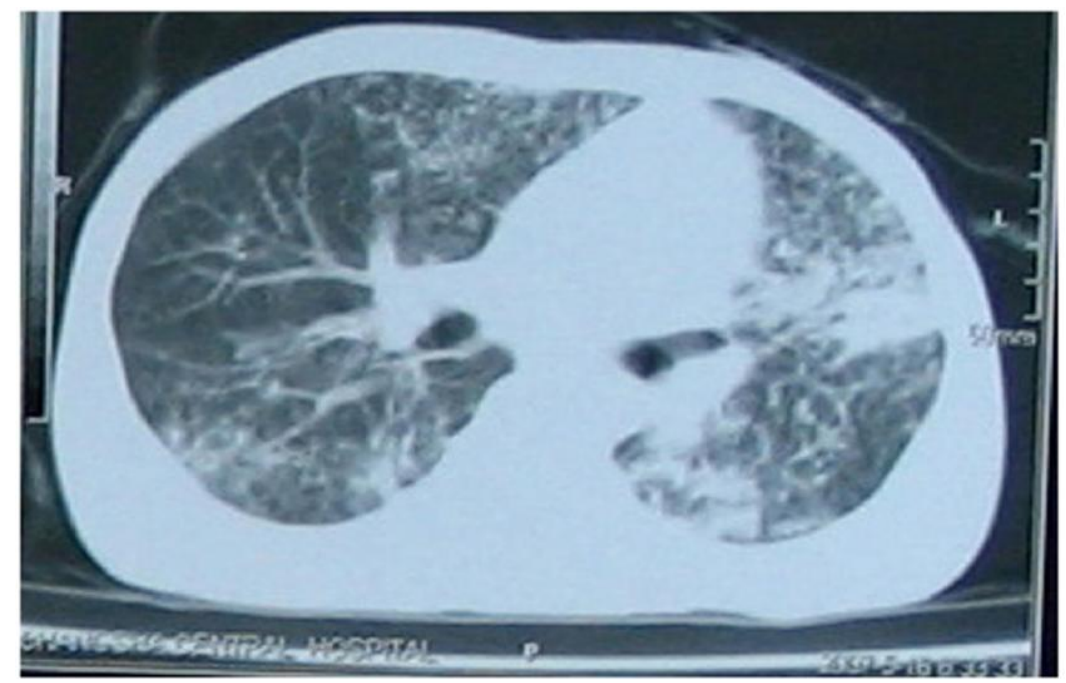

Figure 2. The foci were reduced by treatment.

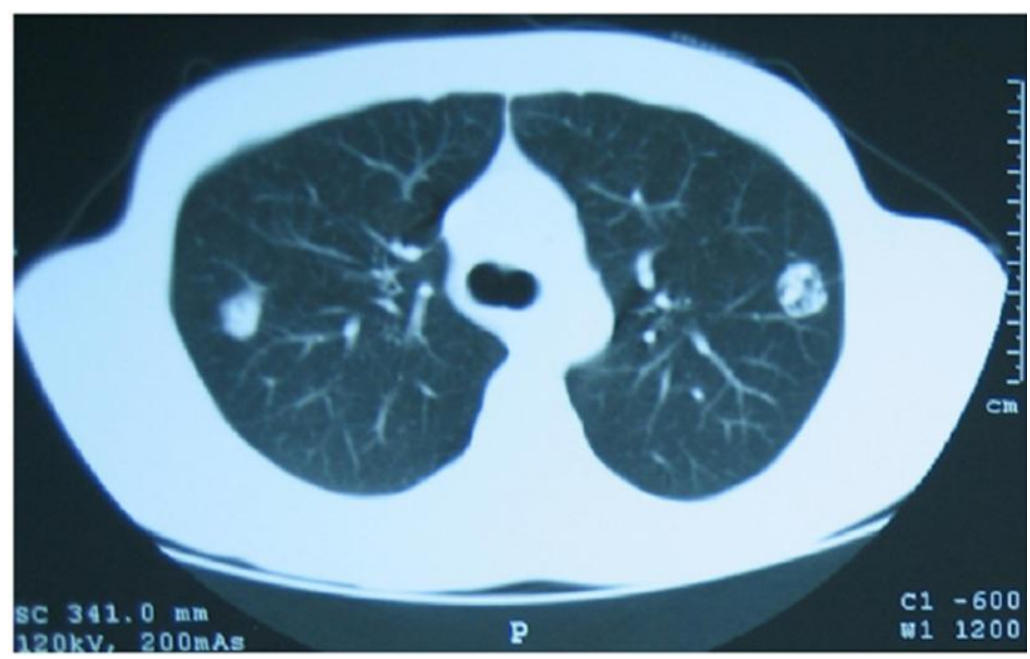

Figure 3. CT shows high density nodules whose size was between 6 to 15 $\mathrm{mm}$; and an elliptical, edge smooth, in homogenous density in them.

magnetic resonance imaging (MRI) examination, while two cases showed normal results, seven cases showed enhancement of the meninges, and one case developed lacunar infarction.

\section{Treatment}

The patients were treated with intravenous $A M B$ combined with fluconazole (FCZ) and intrathecal $A M B$. At the same time, the increased intracranial pressure was reduced through dewatering, nutrition nerve and supporting symptomatic treatment. The AMB was diluted with $500 \mathrm{ml}$ of $5 \%$ glucose prior to administration. The AMB was started at $1 \mathrm{mg} / \mathrm{d}$, then progressively increased to $5 \mathrm{mg} / \mathrm{d}$ daily until it reached $1 \mathrm{mg} /(\mathrm{kg} \cdot \mathrm{d})$; the total dosage was 3 to $5 \mathrm{~g}$. The intrathecal AMB was started at $0.1 \mathrm{mg}$, and then increased by 0.5 to $1 \mathrm{mg}$ daily until the total dosage reached $20 \mathrm{mg}$, and this was repeated twice every week. FCZ was administered at $200 \mathrm{mg}$ daily.
Liver function, renal function, routine blood test, electrolyte, electrocardiogram and cerebrospinal fluid-related items were periodically checked during the treatment period.

\section{RESULTS}

The standard of cure was given as the disappearance of the clinical symptoms, testing negative in the India ink staining, and positive culture for the cerebrospinal fluid. Treatment improvement is the amelioration of the clinical symptoms and the decrease in thalli count, with the culture remaining positive. Treatment inefficacy is the retention or worsening of the clinical symptoms, unchanged thalli count, or death of the patients. Eight 


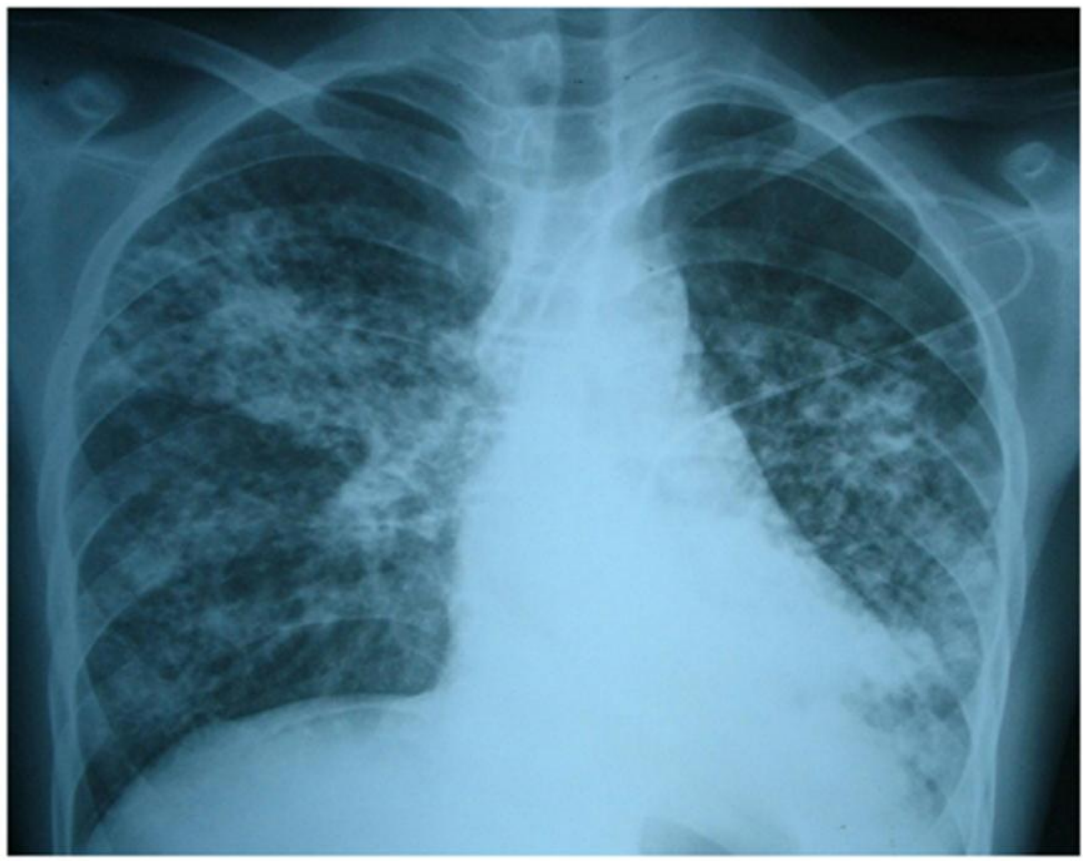

Figure 4. Patchy, nodular, military shadows scattered in two lungs, blurred edges, scattered along lung grain, in homogenous density in them. Some foci blended into clump foci and irregular low density area can be seen in them.

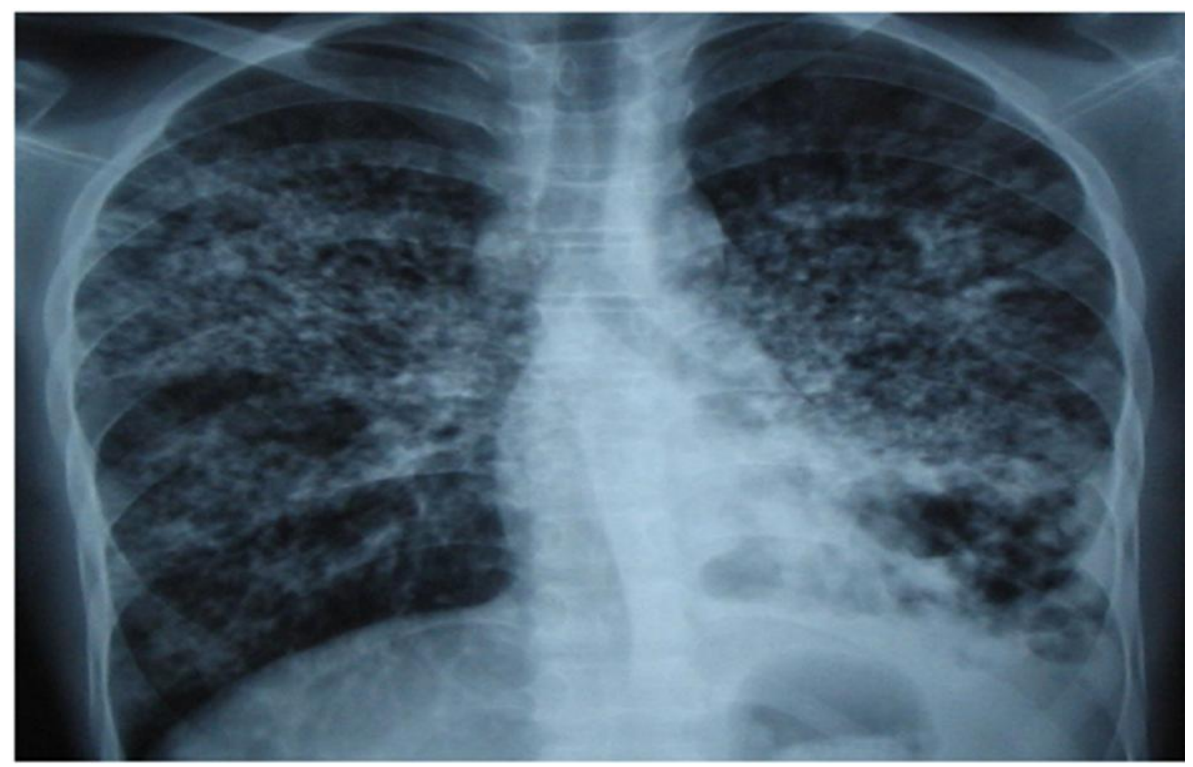

Figure 5. The foci were reduced by treatment.

cases were cured, whereas the remaining four cases were improved. Follow-up at 1 to 24 months $(6.8 \pm 9.5$ months) showed no relapses among the cured patient. Visual acuity declined in one case and the hearing acuity declined in another. The patients were able to work normally and returned to their normal life.

\section{DISCUSSION}

C. neoformans can be found everywhere, in fruits, milk, soil, vespiary, some grasses and plants, human skin and mucosa, pigeon excrement and urine, and in other birds. Birds are one of the main sources of human infections. $C$. 
neoformans is a conditional pathogenic bacterium. In most reported cases, cryptococcosis attacks patients with AIDS, lymphadenosarcoma, Hodgkin's disease, diabetes mellitus, tuberculosis, leukocythemia, renal disease, systemic lupus erythematosus, and so on. A total of $65 \%$ of 37 cases of HIV-negative cryptococcosis reported by Wirojtananugoon have underlying disease, $41 \%$ used immunosuppressants and $16 \%$ suffered from SLE (Kiertiburanakul et al., 2010; Perfect et al., 2010). Similar to the report by Wirojtananugoon, the patient group was HIV negative, with no exposure to birds. However, most of them had chronic consumptive diseases such as chronic viral hepatitis $B$, pneumosilicosis with concurrent tuberculosis, systemic lupus erythematosus, malnutrition, and so on. These consumptive diseases may have disturbed their immune system.

The respiratory tract is generally considered as the invasion gateway of $C$. neoformans. Foci are formed in the lungs that spread through the bloodstream. However, the first symptoms exhibited in this group of patients were headaches, fever and signs of meningeal irritation. Only four cases had respiratory symptoms. This probably relates to the variety of clinical manifestation of PC infections. No symptoms of PC were found and the anomalies were only revealed during plain chest radiography examination. The onset of chronic PC often presents productive cough, chest pain, fever, and so on. Many examinations would not reveal the anomaly. Acute $\mathrm{CP}$ shows a serious lower respiratory tract infection and acute respiratory failure (Shao et al., 2004; Luo et al., 2009; Zhang et al., 2009; Liu et al., 2011). The patients in the present study did not show symptoms of CP.

Therefore, CM patients should undergo lung imaging examinations regardless of the presence of respiratory tract symptoms and physical signs to identify whether or not concurrent pulmonary cryptococcosis exists. Further etiologic examinations should be performed to arrive at a definitive diagnosis. Most of the patients had symptoms of meningitis and physical signs. The diagnosis of $\mathrm{CM}$ was relatively easy through repeated India ink staining and cerebrospinal fluid cultures. However, the diagnosis of PC was relatively difficult because only $20 \%(2 / 10)$ of the patients had symptoms with no obvious physical signs. In this case, the chest imaging examination was very important in the diagnosis. The chest imaging mainly showed five characteristic changes. The chest X-ray showed scattered patchy, nodular shadows, cavitation, hilar lymph node enlargement and interstitial lung changes. Correspondingly, the CT showed highly dense, widespread patchy and speckled shadows, cavities with varying sizes, elliptical, smooth-edged highly dense nodules, enlarged mediastinal lymph nodes, and thickening of the interlobular septa.

Shao et al. (2004) reported that nodular shadows are the most common sign (16/21 cases) of PC, followed by solitary lesions (11/21 cases). Cavities (1/21cases) and diffuse mixed pathologies (2/21 cases) are rare (Zhang et al., 2009). Solitary lesions were comparatively rare in the patient group, whereas cavities were the most common. The variations in the lung imaging results are likely from the combined action of the quantity and virulence of the pathogenic fungi and the body's immunity. The serious patients suffered damaged to lung tissues. The imaging showed diffuse multiple nodular shadows with patchy shadows that even formed cavities. The less serious patients had nodular shadows with decreased cavities and the tendency of forming solitary foci. With antifungal treatment, the foci were clearly reduced. The prognosis of C. neoformans lung infections is usually better among patients with normal immunologic function. Monitoring of the imaging changes is a reliable index for the development and treatment evaluation of this disease (Chang et al., 2006).

PC is easily misdiagnosed (Shirley and Baddley, 2009). The patients were all initially misdiagnosed. The reasons for the misdiagnosis are as follows: first, etiologic examinations for cryptococcosis usually have low sensitivity; second, the knowledge of clinicians on PC imaging, especially in distinguishing it from tuberculosis and lung cancer, should be improved. The main imaging characteristic of PC in individuals with normal immunologic function is the visualization of the lung nodes or bossing, which is usually 5 to $52 \mathrm{~mm}$ in diameter, with a majority of lesions smaller than $10 \mathrm{~mm}$. A total of $80 \%$ of the foci was distributed mainly at the left superior lung in the periphery. Multiple nodes were mostly distributed bilaterally, with most of the nodes having clear edges. Lymphadenitis, mutation, pleural seepage, pleural effusion, air bronchogram and doughnut signs was rare. The occurrence of cavity was also rare in some reports, occurring in about 30 to 40\% (Fox and Muller, 2005; Lindell et al., 2005; Kishi et al., 2006; Lu et al., 2009). However, the CT of the surroundings of lung cancer always shows solitary lung nodes or bossing and may have signs of lobulation, spicule sign, vessel convergence sign, non-fitting eccentric cavity wall, pleural indentation, and so on. These signs were different in the PC. Tuberculosis usually occurs in the posterior segment of the upper lobe of the lungs. Cavitation, fibrosis, calcification and enlargement of the mediastinal lymph nodes were often seen. When the imaging is not easy to differentiate, PC patients should be aware of minor respiratory tract symptoms and the inefficiency of treatment with antibiotics (Song et al., 2010; Wang et al., 2011). Pathologic tissue examinations of percutaneous lung biopsies is necessary (Hu et al., 2006; Zeng et al., 2011).

The up-to-date manual for $C$. neoformans management of infectious disease association (Saag et al., 2000; Shirley and Baddley, 2009; Perfect et al., 2010) suggested that the recommended treatment of non-HIVinfected and non-organ transplantation cryptococcal meningitis patients is amphotericin $B$ deoxycholate $(\mathrm{AmBd})$ administration from 0.7 to $1.0 \mathrm{mg} /(\mathrm{kg} \cdot \mathrm{d})$ 
combined with flucytosine $[100 \mathrm{mg} /(\mathrm{kg} \cdot \mathrm{d})$, taken orally four times], with induction treatment for at least four weeks. Treatment is induced in meningocephalitis patients for four weeks if no nervous system complications occur and cerebrospinal fluid culture is positive after two weeks of treatment. The side effects of $\mathrm{AmBd}$ are significant; therefore, it may be replaced with a lipid formulation of amphotericin B (LFAmB) after two weeks of treatment. Induction of treatment needs to be prolonged to 6 weeks, including the LFAmB treatment for at least 4 weeks, in patients with nervous system complications.

The consolidation treatment with fluconazole ( $400 \mathrm{mg} / \mathrm{d})$ should be then started (B-II). If patients can no longer bear the transfusion of $A m B d$, the $A m B d$ liposome [3 to $4 \mathrm{mg} /(\mathrm{kg} \cdot \mathrm{d})$ ] or ABLC [5 mg/(kg.d)] (B- II) can be used. If flucytosine is not used in treatment induction or suspension, the AmBd- or LFAmB-induced treatment should be prolonged for at least 2 weeks (B-II). If there is little chance of treatment failure (such as, early stage diagnosis, no other diseases or state of immunologic suppression, good curative effect with the combination antifungal treatment for 2 weeks initially), the induced treatment of $\mathrm{AmBd}$ combined with flucytosine can be reduced to 2 weeks, and the sequential fluconazole treatment $\{800 \mathrm{mg} \quad[12 \mathrm{mg} /(\mathrm{kg} \cdot \mathrm{d})$, taken orally]\} for eight weeks. After treatment induction and consolidation, fluconazole treatment [200 mg $(3 \mathrm{mg} / \mathrm{kg}) / \mathrm{d}$, taken orally] can be continued from 6 to 12 weeks (B-II).

The up-to-date manual for $C$. neoformans management of infectious disease association suggested that the recommended treatment for noncentral nervous system cryptococcosis for mild or moderate $C$. neoformans lung infection in non-immunologically suppressed patients is fluconazole $(400 \mathrm{mg} / \mathrm{d}$, taken orally) for 6 to 12 weeks. The continuous positive valence of $C$. neoformans serum antigen is not the standard for continual treatment (B-II). The treatment of severe pneumonia is the same as the treatment of central nervous system infection (B-II). No fluconazole or fluconazole is contraindicated, such as itraconazole (200 mg, twice daily, taken orally), voriconazole ( $200 \mathrm{mg}$, twice daily, taken orally) or posaconazole ( $400 \mathrm{mg}$, twice daily, taken orally) can be used for treatment (B-II). Surgery is suitable for patients who are definitively diagnosed, with continual abnormities in the imaging, and unavailability of antifungal treatment (B-II). Patients with pulmonary cryptococcosis and patients with normal immune systems need to undergo lumbar puncture to exclude external asymptomatic central nervous system infections. However, patients without symptoms of lung nodes or effusion, non-central nervous system symptom, or low or positive valence of $C$. neoformans serum antigen cannot undergo the lumbar puncture (B-II).

Ying et al. (2005) considered fluconazole as an effective pulmonary cryptococcosis treatment and surgery is very effective in a limited number of CP cases (Wang et al., 2005; Liu and Jiang, 2006). A total of 12 cases received the combined $A M B$ and fluconazole treatment. Eight cases were cured and the remaining four cases improved. The effectiveness was $100 \%$. AMB combined with fluconazole is an effective method for treating $\mathrm{CM}$ with concurrent PC.

\section{ACKNOWLEDGEMENT}

This project was supported by Health Department of Hunan Province of the People's Republic of China foundation (B2006-199).

\section{REFERENCES}

Chang WC, Tzao C, Hsu HH, Lee SC, Huang KL, Tung HJ, Chen CY (2006). Pulmonary cryptococcosis: comparison of clinical and radiographic characteristics in immunocompetent and immunocompromised patients. Chest 129(2):333-340.

Fox DL, Muller NL (2005). Pulmonary cryptococcosis in immunocompetent patients: CT findings in 12 patients. AJR. Am. J. Roentgenol. 185(3):622-626.

Hu C, Liu R, Xiao B, Zhang C, Li Y, Tang W, Liang J (2006). The clinical significance of the cerebrospinal fluid examination in cryptococcal meningitis. J. Brain Nerv. Dis. 18(3):183-187.

Hu HJ, Zhou P, Hu JB, Deng LP, Zhang SZ, Ying KJ (2006). A report of 12 cases of primary pulmonary cryptococcosis. Zhonghua Nei Ke Za Zhi 45(9):721-724.

Kiertiburanakul S, Wirojtananugoon S, Pracharktam R, Sungkanuparph $S$ (2010). Cryptococcosis in human immunodeficiency virus-negative patients. Int. J. Infect. Dis. 10(1):72-78.

Kishi K, Homma S, Kurosaki A, Kohno T, Motoi N, Yoshimura K (2006). Clinical features and high-resolution CT findings of pulmonary cryptococcosis in non-AIDS patients. Respir. Med. 100(5):807-812.

Lai G, Lin Q'an, Liu D (2005). Domestic retrospective analysis of pulmonary cryptococcosis in the recent 22 years. Chin J. Pract. Intern. Med. 25(2):176-178.

Lindell RM, Hartman TE, Nadrous HF, Ryu JH (2005). Pulmonary cryptococcosis: CT findings in immunocompetent patients. Radiology 236(1):326-331.

Liu M, Jiang GN (2006). Surgical treatment of pulmonary cryptococcosis. Zhonghua Jie He He Hu Xi Za Zhi, 29(5):307-309.

Liu YN, She DY, Sun TY, Tong ZH, He B, Xiao Y, He LX, Qu JM, Liu $X Q$, Li ER, Chen P, Ma ZS, Shi Y, Feng YL, Jiang SJ, Xiong SD, Hu CP (2011). A multicentre retrospective study of pulmonary mycosis clinically proven from 1998 to 2007. Zhonghua Jie He He Hu Xi Za Zhi 34(2):86-90.

Liu ZY, Wang AX, Li TS (2002). A clinical study of 26 cases of cryptococcal meningitis. Chin. J. Intern. Med. 41(8):543-541.

Lu SH, Hou YY, Tan YS, Liu T, Wang Q, Bai CX (2009). Clinicopathological analysis of primary pulmonary cryptococcosis. Zhonghua Jie He He Hu Xi Za Zhi 32(6):430-433.

Luo YC, Wang Q, Li CC (2009). Highlights of diagnosis and treatment of pulmonary cryptococcosis. Zhonghua Er Ke Za Zhi, 47(3):231-232.

Perfect JR, Dismukes WE, Dromer F, Goldman DL, Graybill JR, Hamill RJ, Harrison TS, Larsen RA, Lortholary O, Nguyen MH, Pappas PG, Powderly WG, Singh N, Sobel JD, Sorrell TC (2010). Clinical practice guidelines for the management of cryptococcal disease: 2010 update by the infectious diseases society of America. Clin. Infect. Dis. 50(3):291-322.

Saag MS, Graybill RJ, Larsen RA, Pappas PG, Perfect JR, Powderly WG, Sobel JD, Dismukes WE (2000). Practice guidelines for the management of cryptococcal disease. Infectious Diseases Society of America. Clin. Infect. Dis. 30(4):710-718.

Shao J, Shi JY, You ZQ, Li TN (2004). CT appearance of pulmonary cryptococcosis. Chin. J. Radiol. 38:831-833. 
Shirley RM, Baddley JW (2009). Cryptococcal lung disease. Curr. Opin. Pulm. Med. 15(3):254-60.

Song KD, Lee KS, Chung MP, Kwon OJ, Kim TS, Yi CA, Chung MJ (2010). Pulmonary cryptococcosis: imaging findings in 23 non-AIDS patients. Korean J. Radiol. 11(4):407-416.

Wang T, Sun YE, Yu CH, Yang B, Sun K, Zhou ZH (2005). Surgical treatment of primary pulmonary cryptococcosis. Zhonghua Wai Ke Za Zhi, 43(22):1447-1449.

Wang YC, Huang ZG, Shi J, Zhang XZ (2011). Diagnostic value of computed tomography in invasive pulmonary fungal infections. Zhonghua Yi Xue Za Zhi 91(1):20-22.

Wei P, Li HP (2007). Clinical analysis of 41 cases of pulmonary cryptococcosis. Chin. J. Mycol. 2(5):267-269.

Xu LY, Liu DL, Yu YH, Wang TZ, Liu DM, Lai GX (2011).Pulmonary cryptococcosis: analysis of 38 cases. Zhonghua Jie $\mathrm{He} \mathrm{He} \mathrm{Hu} \mathrm{Xi} \mathrm{Za}$ Zhi 34(9):653-656.
Ying KJ, Jiang LB, Chen EG, Hong WJ, Jin M (2005). Pulmonary cryptococcosis: analysis of nine cases. Zhonghua Jie $\mathrm{He} \mathrm{He} \mathrm{Hu} \mathrm{Xi} \mathrm{Za}$ Zhi 28(7):464-467.

Zeng Y, Wu X, Yi X, Luo B, Zhu M, Rui W, Zhu X, Li X, Li H (2011). Clinicopathologic and ultrastructural study of non-HIV-related primary pulmonary cryptococcosis in China: report of 43 cases. Ultrastruct. Pathol. 35(1):19-25.

Zhang PH, Hu BJ, He LX, Li HY, Wang BQ, Chen XH, Pan J, Jiang HN, Zhou CM, Gao XD, Xie HM, Huang SL, Xia WS, Tao LL, Bai CX (2009). The characteristics of CT imaging and diagnosis of pulmonary cryptococcosis in 42 cases with non-acquired immune deficiency syndrome. Zhonghua Nei Ke Za Zhi 48(5):362-366. 